

\title{
CONOCIMIENTOS, PERCEPCIONES Y PRÁCTICAS EN PROFESIONALES DE LA SALUD SOBRE MOVILIZACION TEMPRANA EN LOS PACIENTES DE LAS UNIDADES DE CUIDADOS INTENSIVOS
}

\section{KNOWLEDGE, PERCEPTIONS AND PRACTICES IN HEALTH PROFESSIONALS ON EARLY MOBILIZATION IN PATIENTS IN INTENSIVE CARE UNITS}

Ángela Yaleska Arévalo Tabares ${ }^{1}$ https://orcid.org/0000-0001-9690-1038, Freiser Eceomo Cruz Mosquera ${ }^{2}$ https://orcid.org/00000001-7584-4636, Yuly Viviana Valencia Salazar ${ }^{3}$ https://orcid.org/0000-0001-9669-9634, Diego Alonzo Peláez Domínguez ${ }^{3}$ https://orcid.org/0000-0002-7066-289X, Juan David Rosero Portocarrero ${ }^{3}$ https://orcid.org/0000-0003-2453-1351

${ }^{1}$ Terapeuta Respiratoria, Especialista en Rehabilitación Cardiopulmonar. Estudiante maestría en Gestión Pública. Docente Facultad de Salud. Universidad Santiago de Cali. Colombia.

${ }^{2}$ Terapeuta Respiratorio, Especialista en Pedagogía y Docencia, Magister en Epidemiología, estudiante Medicina. Docente Facultad de Salud. Universidad Santiago de Cali. Investigador asociado Minciencias, Director Grupo de Investigación en Salud Integral. Colombia.

${ }^{3}$ Terapeuta Respiratoria Universidad Santiago de Cali. Semillero SISARE. Colombia.

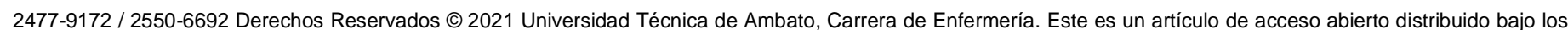


citada.

Recibido: 20 de febrero 2021

Aceptado: 25 de marzo 2021

\section{RESUMEN}

Introducción: Los pacientes críticos son sometidos a intervenciones que traen consigo complicaciones que pueden aparecer incluso de manera precoz, entre ellas se encuentra la debilidad muscular adquirida en unidad de cuidados intensivos, en este contexto tiene una importancia fundamental la movilización temprana del paciente crítico, que facilita el destete de la ventilación mecánica e influye en la calidad de vida posterior al alta. Objetivo: determinar los conocimientos, percepciones y prácticas en profesionales de la salud sobre movilizacion temprana en los pacientes de las unidades de cuidados intensivos de dos centros de salud de Cali. Método: estudio observacional descriptivo de corte transversal en el que se incluyeron 24 profesionales de la salud que laboran en unidades de cuidados intensivos de dos Clínicas de la Ciudad de Cali, a los cuales se les aplicó un cuestionario autoadministrado sobre movilización temprana. El análisis de los datos se realizó en el programa SPSS versión 24 . Resultados: La mayoría de los profesionales de la salud encuestados eran de sexo femenino, con una edad promedio de $31 \pm 5$; se encontró que el $67 \%$ tenía un conocimiento medio, el $25 \%$ bajo y el $8 \%$ conocimiento alto. Por otro lado, el $88 \%$ manifestó ejecutar esta estrategia en su práctica habitual, y reportaron como barreras percibidas ausencia de protocolos y personal insuficiente. Conclusiones: gran parte de los profesionales de salud encuestados mostraron un conocimiento medio sobre movilización temprana y una alta incorporación de esta estrategia a la práctica clínica habitual.

Palabras claves: movilización precoz de paciente crítico, cuidados intensivos, paciente crítico, ventilación mecánica.

\section{ABSTRACT}

Introduction: Critical patients are subjected to interventions that bring complications that can appear even early, among them is muscle weakness acquired in the intensive care unit, in this context, early mobilization of the critical patient is of fundamental importance, which facilitates weaning from mechanical ventilation and influences post-discharge quality of life. Objective: to determine the knowledge, perceptions and practices in health professionals about early mobilization in patients in the intensive care units of two health centers in Cali. Method: a descriptive, cross-sectional observational study that included 24 health professionals working in intensive care units of two Clinics in the City of Cali, to which a self-administered questionnaire on early mobilization was applied. Data analysis was performed using SPSS version 24. Results: Most of the health professionals surveyed were female, with a mean age of $31 \pm 5$; It was found that $67 \%$ had medium knowledge, $25 \%$ low and $8 \%$ high knowledge. On the other hand, $88 \%$ stated that they executed this strategy in their usual practice, and reported the absence of protocols and insufficient personnel as perceived barriers. Conclusions: a large part of the health professionals surveyed showed a medium knowledge about early mobilization and a high incorporation of this strategy into routine clinical practice.

Keywords: early mobilization of critical patients, intensive care, critical patients, mechanical ventilation 
Autor de correspondencia: Mg. Freiser Eceomo Cruz Mosquera. Correo electrónico: freiser.cruz00@usc.edu.co

\section{INTRODUCCIÓN}

En los últimos 30 años las Unidades de Cuidados Intensivos (UCI) han pasado a formar una parte esencial de todos los centros hospitalarios, su impacto en la sobrevida de los pacientes cuya probabilidad con frecuencia es baja, hace que su utilidad sea incuestionable (1-4); sin embargo, la gravedad de los enfermos abordados en este contexto, exige la ejecución de intervenciones complejas que generan complicaciones, que pueden aparecer incluso en las primeras horas de su instauración (5-7).

Intervenciones como la ventilación mecánica y el uso de sedantes llevan al reposo prolongado, dando origen a alteraciones de diferentes sistemas, principalmente el cardiovascular y osteomuscular. Al respecto Convertino et al., evidenciaron que los pacientes al estar confinados a una cama y con limitación de la actividad física presentan disminución del gasto cardiaco, consumo de oxígeno, masa muscular y densidad ósea (8). Además, los pacientes internados en cuidados intensivos experimentan alteraciones metabólicas en las primeras 24 horas de inmovilización, incrementando la probabilidad de desarrollar debilidad muscular adquirida en UCI (9-13). Por las repercusiones que trae esta condición no solo durante la estancia hospitalaria sino posterior al egreso, se han planteado distintas estrategias para evitar su aparición entre las cuales se encuentra la movilización temprana (MT) (14-16). La ejecución de la MT en el paciente crítico y los beneficios que genera han sido ampliamente descritos y respaldados por diferentes investigaciones.

En una revisión sistemática realizada por Adler y Malone, encontraron entre los beneficios asociados a la MT incremento de la fuerza muscular de extremidades superiores e inferiores independiente de los días de ventilación mecánica y en el periodo de rehabilitación mayor distancia caminada, independencia funcional y menor frecuencia de disnea (17). Por otro lado, Naisahdh et al., en una población de 104 pacientes ventilados mecánicamente a quienes se les aplicó un protocolo de movilización temprana consistente en interrupción diaria de la sedación, terapia física y ocupacional, reportaron una mayor independencia funcional en el $59 \%$ de los pacientes y menor tiempo de requerimiento de soporte ventilatorio (18).

A pesar de los beneficios relacionados con la movilización temprana, se ha evidenciado que la aplicación de esta estrategia por circunstancias inherentes al estado del paciente y el conocimiento de los profesionales de salud en algunos casos es limitada. En un estudio realizado en Alemania, se encontró que sólo al $24 \%$ de los pacientes que ingresan a una $\mathrm{UCl}$ se les realiza movilización fuera de la cama, el tipo de paciente en quien menos se ejecuta la intervención es el intubado $8 \%$, traqueostomizados $39 \%$ y ventilados de forma no invasiva 53\%; además, la sedación se identificó como una de las barreras más comunes para realizar la intervención (19). Por otra parte, en una investigación ejecutada en Australia, a partir de la aplicación de una encuesta a 111 profesionales de salud, se informó que habitualmente no se inicia la MT por la presencia de tubo endotraqueal $43 \%$, baja expectativa de tolerancia al ejercicio $20 \%$, requerimiento de mayor personal para la intervención $9 \%$, presencia de sedación y falta de cooperación $8 \%(20)$.

En Estados Unidos, Truong et al., describieron que, si bien la movilización temprana es una práctica trascendental, los protocolos de la $\mathrm{UCl}$ como no manipular con frecuencia el paciente crítico y perseguir resultados inmediatos, son las barreras principales a la hora de ejecutar esta estrategia (21); adicionalmente, cuando se ejecuta las acciones son insuficientes 0 no se hacen con la rigurosidad necesaria. En consonancia con lo anterior, un estudio realizado en Colombia describió que a pesar de la existencia de guías internacionales que incluyen la movilización en sus recomendaciones sobre el manejo de pacientes críticos y las múltiples publicaciones de protocolos de intervención, las estrategias de movilización no se han integrado de manera efectiva a la práctica diaria (22). Por estas razones, se desarrolló la presente investigación con el objetivo de determinar los conocimientos, percepciones y prácticas en profesionales de la salud sobre movilizacion temprana en los pacientes de las unidades de cuidados intensivos de dos centros de salud de Cali.

\section{MÉTODOS}

\section{Diseño y participantes.}

Estudio observacional descriptivo de corte transversal realizado en el segundo semestre del año 2018, en el que se incluyeron 24 profesionales de la salud (Terapeutas Respiratorios y Fisioterapeutas) que laboraban en las unidades de cuidados intensivos de dos instituciones de salud de la Ciudad de Santiago de Cali, Colombia y aceptaron participar voluntariamente de la investigación, se excluyeron los participantes que elaboraron de manera incompleta el instrumento de recolección de la información.

De cada participante se registraron variables sociodemográficas y profesionales tales como edad, sexo, profesión, realización de posgrados clínico, experiencia laboral, experiencia en $\mathrm{UCl}$, tipo de unidad de cuidados intensivos y educación continua en MT, para establecer el conocimiento se usaron variables categóricas como consecuencias del reposo prolongado, actividades consideradas en el marco de la MT, indicaciones y contraindicaciones principales de la movilización, escalas de valoración y complicaciones. En relación con la percepción y ejecución de la MT, se usaron variables: tiempo de inicio de la movilización, criterios de inicio, actividades con las que integra la MT, barreras asociadas al paciente, al personal y la institución de salud.

Las variables mencionadas se consolidaron en un cuestionario autoadministrado, validado por expertos especialistas en rehabilitación cardiopulmonar, el cual fue entregado a cada profesional. El cuestionario estaba constituido por 20 ítems de los cuales 6 relacionados con aspectos sociodemográficos y profesionales, 7 ítems evaluaban el conocimiento y 7 ítems las percepciones y prácticas sobre movilización temprana. El conocimiento se dividió en bajo, medio o alto de acuerdo con el número de preguntas contestadas de manera adecuada (1-3, 4-5, 6-7 respectivamente). 


\section{Análisis estadístico.}

A partir de la información registrada en la base de datos de Excel 2007 se realizó el análisis estadístico en el Programa IBM SPSS versión 24. Inicialmente se estableció la normalidad de la distribución de las variables cuantitativas usando la prueba de Shapiro Wilk, las variables categóricas se expresaron como valor frecuencia y porcentaje.

\section{RESULTADOS}

La mayor parte de los encuestados eran de sexo femenino $58 \%$, con una edad promedio de 31 años \pm 5 , el $96 \%$ eran profesionales en Fisioterapia, tenían más de un año de haber egresado y un 29\% había desarrollado una especialización clínica. Por otro lado, el 96\% manifestó tener más de un año de experiencia laboral, y el $50 \%$ entre 1 y 5 años de experiencia

\section{Aspectos éticos.}

La investigación siguió los lineamientos éticos de la resolución 8430 del Ministerio de Salud de Colombia y de la Declaración del Helsinki; el protocolo contó con el aval del Comité de ética de la Universidad Santiago de Cali y las instituciones en la que se ejecutó la investigación.

en unidad de cuidados intensivos. Con relación al tipo de $\mathrm{UCI}$ en la cual laboran el $88 \%$ manifestó hacerlo en unidades médico-quirúrgicas, el $8 \%$ en unidad cardiovascular y el $4 \%$ en unidad neurológica. Referente a el número de pacientes atendidos por turno, ningún encuestado refirió atender menos de 5 pacientes, el $62,5 \%$ atienden entre $6-10$ y el $37,5 \%$ más de $10 \quad$ (Tabla 1).

\section{TABLA1.}

CARACTERÍSTICAS SOCIODEMOGRÁFICAS, PROFESIONALES Y LABORALES DE LOS ENCUESTADOS.

\begin{tabular}{|c|c|c|c|}
\hline \multicolumn{2}{|c|}{ Aspecto } & \multirow{2}{*}{$\begin{array}{c}\begin{array}{c}\text { Frecuencia } \\
\mathbf{N}^{\circ}\end{array} \\
10\end{array}$} & \multirow{2}{*}{$\begin{array}{c}\begin{array}{c}\text { Porcentaje } \\
\%\end{array} \\
42\end{array}$} \\
\hline Sexo & Masculino & & \\
\hline & Femenino & 14 & 58 \\
\hline Total & & 24 & 10 \\
\hline \multirow[t]{2}{*}{ Profesión } & Terapia respiratoria & 1 & 4 \\
\hline & Fisioterapia & 23 & 96 \\
\hline Total & & 24 & 100 \\
\hline \multirow[t]{2}{*}{ Posgrado } & Especialización & 7 & 29 \\
\hline & Ninguno & 17 & 71 \\
\hline Total & & 24 & 100 \\
\hline \multirow[t]{3}{*}{ Experiencia laboral } & $<1$ año & 1 & 4 \\
\hline & 1-5 años & 10 & 42 \\
\hline & $>5$ años & 13 & 54 \\
\hline Total & & 24 & 100 \\
\hline \multirow[t]{3}{*}{ Experiencia en UCI } & $<1$ año & 3 & 13 \\
\hline & 1-5 aก็ัง & 12 & 50 \\
\hline & $>5$ años & 9 & 37 \\
\hline Total & & 24 & 100 \\
\hline \multirow[t]{4}{*}{ Tipode UCI } & Médico-quirúrgica & 21 & 88 \\
\hline & Trauma & o & o \\
\hline & Neurològica & 1 & 4 \\
\hline & Cardiovascular & 2 & 8 \\
\hline Total & & 24 & 100 \\
\hline \multirow[t]{3}{*}{ Pacientes atendidos por turno } & $1-5$ & o & o \\
\hline & $6-10$ & 15 & 62,5 \\
\hline & $>10$ & 9 & 37,5 \\
\hline Total & & 24 & 100 \\
\hline
\end{tabular}

Al evaluar el nivel de conocimiento de movilización temprana, se evidenció que el $63 \%$ reconocía todos los aspectos que involucra la definición, por otro lado, el 58\% conocía alguna escala de evaluación funcional; con relación al momento de inicio de la MT el 50\% manifestó en que dependía del estado clínico del paciente y el resto consideraban que debía iniciarse entre 24-48 horas. En coherencia con lo anterior, el 54\% de los sujetos estudiados coinciden en el hecho de que la principal indicación para el inicio es que el paciente esté clínicamente estable. En este segmento es importante resaltar que el 26\% consideran erróneamente que la MT se debe circunscribir a los pacientes con reposo prolongado y sospecha de debilidad muscular. Al indagar sobre las consecuencias del reposo prolongado en $\mathrm{UCl}$ el $71 \%$ de la población las reconoció a cabalidad. Para finalizar, con relación a las contraindicaciones para el inicio de la MT el 46\% logró identificarlas de manera cabal y respecto a las complicaciones derivadas de esta estrategia, el 67\% contestó inestabilidad hemodinámica y el 
$33 \%$ desconexión de dispositivos como catéteres, sondas o tubos. A partir de lo mencionado, se estimó que sólo el $8 \%$ tiene un nivel alto conocimiento sobre la estrategia de movilización temprana, $25 \%$ nivel alto y $67 \%$ nivel medio (Figura1).

FIGURA 1.

NIVEL DE CONOCIMIENTO SOBRE MOVILIZACIÓN TEMPRANA

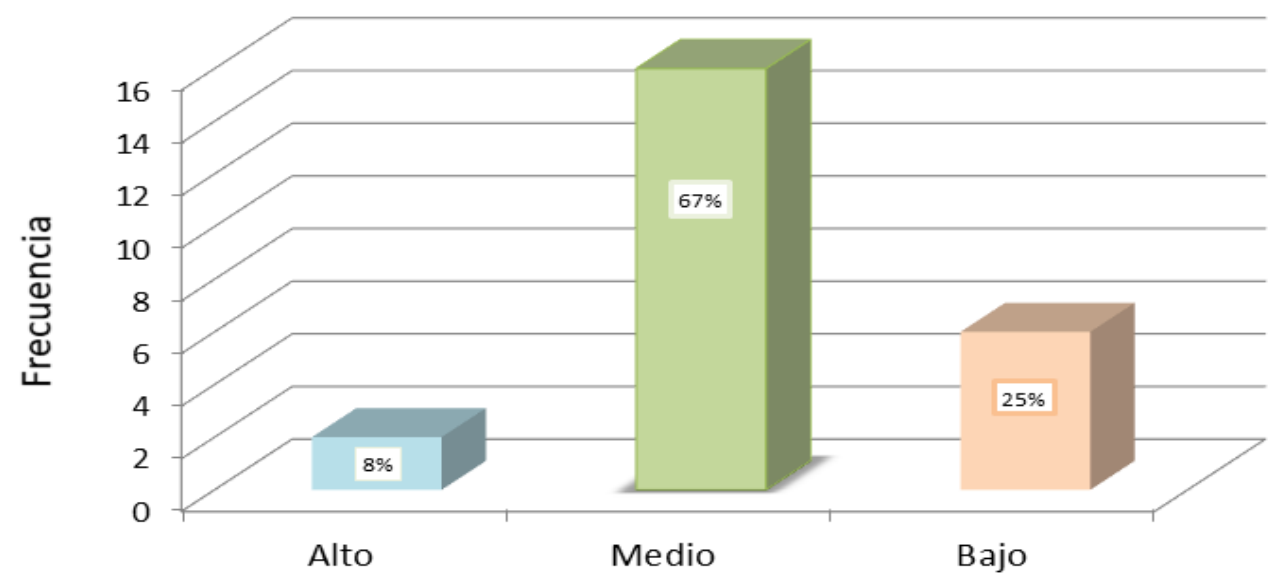

clínico, sobre la ejecución de intervenciones simultáneas a la $\mathrm{MT}$, el $48 \%$ refirió que no integra esta práctica con otra

Con relación a la caracterización de la práctica de movilización temprana $88 \%$ profesionales de salud ejecutaron esta intervención en los pacientes que ingresan a UCI, 19 (90\%) intervención y el 38\% manifestó que la integra con el destete ventilatorio (Tabla 2).

realizaban MT una vez por turno; Por otra parte, respecto a los criterios para iniciar la MT el $71 \%$ refiere que la inicia por juicio

TABLA 2.

CARACTERIZACIÓN DE LA PRÁCTICA DE MOVILIZACIÓN TEMPRANA.

\begin{tabular}{llcc}
\hline & Aspecto & $\begin{array}{c}\text { Frecuencia } \\
\mathbf{N}^{\circ}\end{array}$ & $\begin{array}{c}\text { Porcentaje } \\
\%\end{array}$ \\
\hline \multirow{2}{*}{ ¿Realiza movilización temprana? } & $\mathrm{Si}$ & 21 & 88 \\
& $\mathrm{No}$ & 3 & 12 \\
\hline Total & & 24 & 100 \\
\hline Frecuencia de ejecución MT & 1 vez por turno & 19 & 90 \\
& 1 Vez al día & 1 & 5 \\
& Cada 2 horas & 1 & 5 \\
\hline Total & & 21 & 100 \\
\hline Criterio de inicio de la MT & Orden médica & 1 & 5 \\
& Protocolo & 5 & 24 \\
& Juicio clínico & 15 & 71 \\
\hline Total & & 21 & 100 \\
\hline Actividades con las que integra MT & Interrupción diaria de la sedación & 3 & 14 \\
& Inicio del destete & 8 & 38 \\
& No tiene en cuenta otra intervención & 10 & 48 \\
\hline Total & & 21 & 100 \\
\hline
\end{tabular}

Fuente: instrumento aplicado por los autores. MT: movilización temprana 
Las barreras percibidas por los profesionales de salud que refirieron realizar movilización temprana se dividieron en institucionales, profesionales y asociadas a los pacientes. En primera instancia, las barreras institucionales percibidas con mayor frecuencia son ausencia de protocolos $38 \%$, insuficiencia de equipos $38 \%$ y falta de orden médica $14 \%$; con relación a las barreras del personal salud se destacan personal insuficiente $38 \%$, el hecho de que algunos consideran que la MT no es una prioridad $24 \%$, la ausencia de coordinación de intervenciones en la entrega de turno y el retraso en la identificación de los pacientes que requieren la MT 14\%. Para finalizar, las barreras inherentes a los pacientes fueron inestabilidad clínica $76 \%$ y suministro de excesiva sedación 14\%. (Tabla 3).

TABLA 3.

BARRERAS PERCIBIDAS EN LA INSTITUCIÓN, EL PERSONAL Y LOS PACIENTES PARA REALIZAR MOVILIZACIÓN TEMPRANA.

\begin{tabular}{llcc}
\hline & Barreras percibidas & Frecuencia & Porcentaje \\
& & N $^{\circ}$ & $\%$ \\
\hline Barreras institucionales & Ausencia de protocolos & 8 & 38 \\
& falta de orden médica & 3 & 14 \\
& Insuficiencia de equipo & 8 & 38 \\
& Práctica costosa & 0 & 0 \\
& Espacio insuficiente & 2 & 10 \\
\hline Total & & 21 & 100 \\
\hline Barreras del personal & Personal insuficiente & 8 & 38 \\
& Entrenamiento inadecuado & 2 & 10 \\
& MT no es una prioridad & 5 & 24 \\
& No coordinación en entrega de turno & 3 & 14 \\
& Retraso en la identificación del paciente para MT & 3 & 14 \\
\hline Total & & 21 & 100 \\
\hline Barreras del paciente & Inestabilidad clínica & 16 & 76 \\
& Excesiva sedación & 3 & 14 \\
& Desconexión de dispositivos & 1 & 5 \\
& Alteraciones nutricionales & 0 & 0 \\
& Restricción física & 1 & 5 \\
\hline Total & & 21 & 100 \\
\hline
\end{tabular}

Fuente: instrumento aplicado por los autores. MT: movilización temprana

\section{DISCUSIÓN}

La movilización temprana es una estrategia terapéutica que ha tomado fuerza en las últimas décadas, entre otras cosas porque sus beneficios hasta ahora descritos superan ampliamente los riesgos, siendo algunos de ellos menos días en ventilación, menor estancia en la unidad de cuidados intensivos, menor tiempo de hospitalización y mejor calidad de vida posterior al egreso (23-26). A pesar de lo anterior, no se estudia con la frecuencia esperada los conocimientos, percepciones y prácticas de los profesionales encargados de su ejecución en el escenario clínico.
Con relación al nivel de conocimiento, en este estudio se determinaron los niveles bajo, medio o alto de acuerdo con el número de respuestas acertadas, encontrando que el $75 \%$ de los encuestados tenía un nivel alto o medio y sólo el $25 \%$ un nivel bajo, lo que podría relacionarse con los años de experiencia en cuidados intensivos, dado que el $87 \%$ de los profesionales habían trabajado por más de un año en esta área, similar a la investigación realizada por Curti et al., sugieren que la base del éxito para la atención de los pacientes radica en el conocimiento, dado que este permite generar "modelos mentales" para la ejecución correcta de las intervenciones, constituyendo un argumento para afirmar que una atención pertinente, está determinada por las 
competencias cognitivas y procedimentales del personal de salud relacionada con la movilización temprana (27).

Otros estudios han evaluado el conocimiento sobre la MT, encontrando resultados divergentes a la investigación actual. Al respecto, un estudio realizado por $\mathrm{Koo}$ et al., en la que se incluyeron médicos y fisioterapeutas de 46 universidades de Canadá reportó que el 59,8\% tenía conocimientos insuficientes para movilizar a los pacientes ventilados y el 68\% de los encuestados subestimaron la incidencia de la debilidad muscular adquirida en $\mathrm{UCl}$ (28). De la misma manera , Akinrime et al., en una muestra de 131 profesionales, 44\% médicos, $26 \%$ fisioterapeutas, 30\% enfermeras, evidenciaron que el $89 \%$ de los médicos indicaron tener conocimientos sobre los beneficios de la movilización temprana, pero el $70 \%$ mostró un conocimiento inadecuado sobre lo que constituye esta intervención y apenas el 5\% conocía guías de MT; los datos publicados por los autores mencionados revelan un conocimiento significativamente más bajo que el arrojado en esta investigación (29).

Dentro de las prácticas evaluadas en el estudio actual se estableció que aproximadamente la mitad de los profesionales manifiestan no integrar la movilización con otras estrategias, lo que podría sugerir la no vinculación a la práctica diaria de paquetes de medidas para el tratamiento integral de pacientes graves, es importante resaltar, que desde hace más de 5 años expertos en cuidados intensivos plantearon el "paquete" $\mathrm{ABCDE}$, en el cual reúnen diferentes acciones tales como: evaluación, prevención y tratamiento del dolor, manejo de la sedación, prevención del delirium y movilización precoz, todas orientadas a mejorar las condiciones de los pacientes en estado crítico, principalmente su calidad de vida posterior al egreso $(30,31)$.
Finalmente, las barreras declaradas por los participantes al momento de iniciar la movilización temprana fueron: ausencia de protocolos, equipo de salud insuficiente, no contar con los dispositivos e inestabilidad hemodinámica en los pacientes, estas barreras tienen estrecha relación con lo mostrado en estudios realizados por Caitano et al., y Dubb et al., en los que han descrito limitantes como sedación excesiva, inestabilidad, personal limitado, problemas de seguridad, directrices y equipos insuficientes $(32,33)$. Lo anterior indica que si bien puede haber divergencias entre las investigaciones en términos de conocimientos sobre MT, las limitaciones relacionadas con el paciente y la institución para ejecutar esta estrategia, parecen ser transversales y hacer parte de la percepción de los profesionales de las distintas $\mathrm{UCI}$.

\section{CONCLUSIONES}

Gran parte de los sujetos encuestados eran de sexo femenino, con experiencia superior a un año en el manejo de pacientes críticos y se habían capacitados en la movilización temprana en pregrado o posgrado antes del estudio. Se evidenció elevado porcentaje de los profesionales ejecutan la MT en su práctica diaria, más de la mitad tenía un conocimiento medio sobre esta estrategia y percibía diversas barreras relacionadas con el personal, el paciente y la institución para su realización. La movilización temprana es una estrategia terapéutica que ha tomado fuerza en las últimas décadas, porque sus beneficios superan ampliamente los riesgos, siendo algunos de ellos menos días en ventilación, menor estancia en la unidad de cuidados intensivos, menor tiempo de hospitalización y mejor calidad de vida posterior al egreso

FINANCIAMIENTO: No hubo fuentes de financiamiento.

CONFLICTOS DE INTERÉS: Los autores declaran no tener conflictos de interés.

\section{REFERENCIAS}

1. García C, Martínez C. La realidad de la Unidad de Cuidados Intensivos." Medicina crítica. 2017;31(3):171-173. Disponible en: https://www.medigraphic.com/pdfs/medcri/ti-2017/ti173k.pdf

2. Morales F, Campo C, Arguello M, Salas D. Sobrevida a los 28 días y condición mental y física de los pacientes egresados de una Unidad de Cuidados Intensivos de tercer nivel. Acta méd. Costarric. 2016;58(1):22-26. Disponible en: https://www.scielo.sa.cr/pdf/amc/v58n1/0001-6002-amc-58-01-22.pdf

3. Padilla J. Medicina evolucionista en terapia intensiva: un nuevo paradigma. Acta méd. costarric. 2018;60(1):714.Disponible en: https://www.scielo.sa.cr/pdf/amc/v60n1/0001-6002-amc-60-01-7.pdf

4. Moreira H, Verga F, Barbato M, Burghi. Impacto pronóstico del momento de ingreso y egreso de la unidad de cuidados intensivos. Rev. bras. ter. Intensive. 2017; 29(1):63-69. Disponible en: https://doi.org/10.5935/0103-507x.20170010

5. Sasaki N, Meyer MJ, Eikermann M. Postoperative respiratory muscle dysfunction: Pathophysiology and preventive strategies. Anesthesiology. 2013;118: 961-978. Disponible en : https://doi.org/10.1097/ALN.0b013e318288834f

6. Abdelrazik A, Salah M. Ventilator-associated pneumonia in adult intensive care unit prevalence and complications. The Egyptian Journal of Critical Care Medicine. 2017;5(2):61-63. Disponible en: https://doi.org/10.1016/j.ejccm.2017.06.001

7. Jubran A, Grant B, Duffner L, Collins E, Lanuza D, Hoffman L, et al. Long-Term Outcome after Prolonged Mechanical Ventilation. A Long-Term Acute-Care Hospital Study. Respiratory Research. 2019;22:1:1508-1516. Disponible en: https://doi.org/10.1164/rccm.201806-11310C

8. Convertino A, Bloomfield A, Greenleaf E. An overview of the issues: physiological effects of bed rest and restricted physical activity. Medicine and science in sporrrs and exercice; 1997;29(2):187 -190. Disponible en: 10.1097 / 00005768199702000-00004

9. Pardo J, Ruiz J. Síndrome de desacondicionamiento físico en el paciente en estado crítico y su manejo. Medicina Ac. Col. 2001;23(1):29-34. Disponible en: https://revistamedicina.net/ojsanm/index.php/Medicina/article/view/55-5 
10. Godoy D, Mello L, Masotti L, Napoli M. Intensive Care Unit Acquired Weakness (ICU-AW): a brief and practical review. Reviews in Health Care 2015;6(1):9-35. Disponible en: 10.7175 / rhc.v6i1.1037

11. E Vilas, Fernandez M, Navarro C, Suarez L, Garcia L. Síndrome neuromuscular adquirido en el paciente crítico. Rev Neurol. 2006: 43(11):674-680. Disponible en: https://pesquisa.bvsalud.org/portal/resource/pt/ibc-045638

12. Vanhorebeek I, Latronico N,Berghe V. ICU-acquired weakness. Intensive Care Med.2020;46:637-653. Disponible en: https://doi.org/10.1007/s00134-020-05944-4

13. Stevens D, Marshall S, Cornblath D, Hoke A,Needham D,Jonghe B, et al. A framework for diagnosing and classifying intensive care unit-acquired weakness. Crit Care Med.2009;37(10):S299-308. Disponible en: 10.1097/CCM.0b013e3181b6ef67

14. Gosselink R, Clerckx B, Robbeets C, Vanhullebusch T, Vanpee G, Segers J. Physiotherapy in the intensive care unit. Neth J Crit Care 2011;15(2):66-75. Disponible en: https://www.tkafa.gr/img/enimerosi_files/0408550001415752023100000.pdf

15. Calixto A, Mendoza N, Pérez O, Martínez M, Tomas E, Martínez B. Movilización Temprana Como Prevención Y Tratamiento Para La Debilidad Adquirida En La Unidad De Cuidados Intensivos En Pacientes En Ventilación Mecánica. Experiencia En Un Hospital De Segundo Nivel. European Scientific Journal.2018;14(21):19-30. Disponible en: https://doi.org/10.19044/esj.2018.v14n21p19

16. Zang K, Chen B, Wang M, Chen D, Hui L, Guo S, et al. The effect of early mobilization in critically ill patients: A meta-analysis. Nursing in Critical Care. 2019;25(6):360-367. Disponible en: https://doi.org/10.1111/nicc.12455

17. Adler J, Malone D. Early mobilization in the intensive care unit: a sistematic Review. Cardiopulmonary Physical Therapy Journal.2012;23:5-13. Disponible en: https://www.ncbi.nlm.nih.gov/pmc/articles/PMC3286494/

18. Brahmbhatt N, Murugan R, Milbrandt B. Early mobilization improves functional outcomes in critically ill patients. Critical Care. 2010;14(5):321. Disponible en: https://pubmed.ncbi.nlm.nih.gov/20880413/

19. Nydahl P, Ruhl P, Bartoszek G, Dubb R, Filipovic S, Flohr J et al. Early Mobilization of mechanically ventilated patients: a 1 day point prevalence study in Germany. Critical care medicine. 2014;42(5) :1178-1185. Disponible en: 10.1097/CCM.0000000000000149

20. Skinner E, Berney S, Warrillow S, Denehy L. Rehabilitation and exercise prescription in Australian intensive care units. Physiotherapy. 2008:94(3):220-229. Disponible en: https://doi.org/10.1016/j.physio.2007.11.004

21. Truong A, Fan E, Brower R, Needham D. Bench-to-bedside review: mobilizing patients in the intensive care unit from pathophysiology to clinical trials. Crit Care. 2009;13(4)216. Disponible en: https://www.ncbi.nlm.nih.gov/pmc/articles/PMC2750129/

22. Wilches E, Arcos V, Giron E, Dayana O, Arias M, Pelaez G. Implementación de un programa de movilización temprana en pacientes críticos. Acta Colomb Cuid Intensivo. 2015;15(4):287-292. Disponible en: 10.1016 / j.acci.2015.08.003

23. Taito S, Shime N, Ota K, Yasuda H. Early physiotherapy in the respiratory intensive care unit. Journal of Intensive Care. 2016;4: 50. Disponible en: 10.1186/s40560-016-0179-7

24. Segura D, Martínez V, Herrera Y, Medina C. Movilización temprana, duración de la ventilación mecánica y estancia en cuidados intensivos. Rev. Fac. Med. 2013;61(4):373-379. Disponible en: https://revistas.unal.edu.co/index.php/revfacmed/article/view/42783/44294

25. Chen B, Xiaofang Y, Wanqing L.A systematic review and meta-analysis of the effects of early mobilization therapy in patients after cardiac surgery. Medicine. 2020;99:4(e18843). Disponible en: http://dx.doi.org/10.1097/MD.0000000000018843

26. Ching H, Shing J, Shiang W, Shuo G, Ying W, Yun Y. Early Mobilization of Mild-Moderate Intracerebral Hemorrhage Patients in a Stroke Center: A Randomized Controlled Trial. Neural Repair.2020;34(1)72-81.Disponible en: https://doi.org/10.1177/1545968319893294

27. Curtis J, Cook D, Wall R, Angus D, Bion J, Kacmarek R, et al. ntensive care unit quality improvement: A "how-to" guide for the interdisciplinary team. Critical care medicine.2006;34(1): 218. https://doi.org/10.1097/01.CCM.0000190617.76104.AC

28. Koo k, Choong K, Cook D, Herridge M, Guyatt G, Priestap F, et al. Early mobilization of critically ill adults: a survey of knowledge, perceptions and practices of Canadian physicians and physiotherapists. CMAJ OPEN.2016;4(3):448-453. Disponible en: 10.9778/cmajo.20160021

29. Akinremi A , Ogwu S, Sanya A, Sanusi A, Osinaike B. Early Mobilization in the ICU: A Multicenter Survey of Clinicians' Knowledge, Attitude and Practices in Resource-Limited Hospital Settings. Ann Med Health Sci Res. 2020;10:778-784. Disponible en: https://www.amhsr.org/articles/early-mobilization-in-the-icu-a-multicenter-survey-of-cliniciansrsquoknowledge-attitude-and-practices-in-resourcelimited-hospital-5564.html

30. Morandi A, Piva S, Latronico N. Worldwide ABCDEF (Assessing Pain, Both Spontaneous Awakening and Breathing Trials, Choice of Drugs, Delirium monitoring/management, Early exercise/mobility, and Family Empowerment) Survey. Crit Care Med.2017;45(11):e1111-e1122. Disponible en: :10.1097/CCM.0000000000002640

31. Pandharipande P, Banerjee A, McGrane S,Ely W. Liberation and animation for ventilated ICU patients: the ABCDE bundle for the back-end of critical care. Crit Care.2010; 14:157. Disponible en: https://doi.org/10.1186/cc8999

32. Caitano P, Forgiarini L, Friedman G. Clinical attitudes and perceived barriers to early mobilization of critically ill patients in adult intensive care units. Rev Bras Ter Intensiva. 2018;30(2):187-194. Disponible en: https://www.ncbi.nlm.nih.gov/pmc/articles/PMC6031424/pdf/rbti-30-02-0187.pdf

33. Dubb R, Nydahl P, Hermes C, Schwabbauer N, Toonstra A, Parker A, et al. Barriers and Strategies for Early Mobilization of Patients in Intensive Care Units. AnnalsATS. 2016;13(5):724-730. Disponible en:https://doi.org/10.1513/AnnalsATS.201509-586CME. 